\title{
Factors influencing HIV infection in men who have sex with men in China
}

\author{
Hong-Lv Xu' ${ }^{1}$, Man-Hong Jia ${ }^{2}$, Xiang-Dong Min ${ }^{2}$, Ren-Zhong Zhang ${ }^{2}$, Chun-Jie Yu ${ }^{1}$, Jue Wang ${ }^{2}$, You-Fang Li ${ }^{2}$, \\ Ling Wang ${ }^{2}$, Song-Feng Pan $^{2}$ and Lin $\mathrm{Lu}^{1,2}$
}

To identify risk factors for HIV infection among men who have sex with men (MSM) and to provide a theoretical basis for prevention interventions. Between December 2011 and August 2012, a case-control study was conducted among MSM who underwent voluntary counselling and testing for HIV. Confirmed HIV-positive MSM were included in the case group, and HIV-negative MSM were included in the control group. Information on possible risk factors was collected by a survey questionnaire and a qualitative interview. The results of a conditional logistic regression showed that the following were influencing factors for HIV infection: average monthly income between 2001 and 3000 Yuan (odds ratio $(\mathrm{OR})=6.341,95 \% \mathrm{Cl}$ : 1.714-12.544), only sometimes using condoms when having anal sex with men in the last 6 months (OR=7.601, 95\% Cl: 1.359-23.083), having HIV-positive sex partners (OR=5.273, 95\% Cl: 1.572 $17.691)$, rectal trauma with bleeding in the last 6 months $(\mathrm{OR}=2.947,95 \% \mathrm{Cl}: 1.308-6.638)$, not using condoms at last sexual encounter $(\mathrm{OR}=1.278,95 \% \mathrm{Cl}: 1.012-5.595)$, engaging in commercial sex $(\mathrm{OR}=5.925,95 \% \mathrm{Cl}: 1.923-13.890)$ and having more than 16 sex partners in the last 6 months $(O R=1.175,95 \% \mathrm{Cl}: 1.021-1.353)$. These seven factors were the risk factors of HIV infection (OR $>1)$. However, having anal sex less than 10 times in the previous 1 month $(0 R=0.002,95 \% \mathrm{Cl}: 0.000-0.287)$ was a protective factor against HIV infection among $M S M(O R<1)$, and insertive $(O R=0.116,95 \% \mathrm{Cl}: 0.000-0.236)(O R<1)$ anal intercourse influenced HIV infection. Interventions should be targeted at MSM whose average monthly income is between 2001 and 3000 Yuan, and who engage in commercial sex. In addition, the importance of using condoms at every sexual encounter should be emphasised in health education, as should the treatment of rectal trauma with bleeding. Finally, MSM should decrease the number of sex partners and frequency of anal sex to decrease the rate of HIV infection.

Asian Journal of Andrology (2013) 15, 545-549; doi:10.1038/aja.2013.51; published online 27 May 2013

Keywords: case-control study; HIV infection; influencing factors; men who have sex with men (MSM)

\section{INTRODUCTION}

In recent years, the rate of HIV infection among men who have sex with men (MSM) in China has increased significantly each year. The HIV infection rate is higher than 10\% among MSM in Chengdu and Kunming in southwest China. ${ }^{1-4}$ As a result, growing attention is being given to the risk factors for HIV infection among MSM. Research from China and abroad suggests that the risk factors for HIV infection among MSM are as follows. Unprotected anal sex: a recent study showed that the rates of never using or sometimes using condoms when having same-sex anal intercourse in the last 6 months among MSM in China were 8.6\% (120/1390) and 45.3\% (630/1390), respectively. ${ }^{5}$ A recent report showed that $53.4 \%$ (155/290) of MSM had always used condoms with a male partner in the last 6 months in Nigeria. ${ }^{6}$ Multiple sex partners: the number of sex partners has been shown to positively correlate with HIV infection among MSM.,8 Approximately 60.9\% (390/640) of MSM in Puerto Rico, North America, have had at least 10 sex partners throughout their life, ${ }^{9}$ while in the United States, $76.0 \%(n=8505 / 11191)$ of MSM have had an average of three sex partners. ${ }^{10}$ Sexual behaviour pattern: the main sexual behaviours include anal sex, oral sex and masturbation; however, other acts, such as fisting, are also performed. In America, MSM may also partake in sexual masochism as a sexual behaviour, and this is a risk factor for HIV infection. ${ }^{11-13} \mathrm{HIV}$-positive sex partners: in New York City, the United States, 36.3\% (201/554) of MSM have reported sexual behaviour with an HIV-positive partner before becoming infected, ${ }^{14}$ and in South Africa, having more than one HIV-positive sex partner has been reported as a risk factor. ${ }^{15}$ Sexually transmitted infections: sexually transmitted infections increase the risk of HIV transmission. ${ }^{16}$ HIV/AIDS awareness: MSM have been reported to have very low rates of HIV/AIDS awareness, ${ }^{17}$ primarily in Africa; however, higher awareness rates have been reported in North America. Age: the risk of HIV infection is inversely associated with age at the time of the first sexual encounter with a man. ${ }^{18,19}$ Drug use: drinking and drug use lead to less efficient use of condoms. ${ }^{20-22}$ Income: an average monthly income higher than 300 dollars has been associated with HIV infection among MSM in Nanjing, China. ${ }^{23}$ Although many studies have investigated risk factors for HIV infection among MSM in China, the results are not conclusive and may not be applicable to Yunnan, as China is a multiethnic society with significant differences in living habits, local culture

${ }^{1}$ Kunming Medical University, Kunming, Yunnan 650500, China and ${ }^{2}$ Yunnan Center for Disease Control and Prevention, Kunming, Yunnan 650022, China Correspondence: Dr L Lu (lulin@yncdc.cn)

Received: 19 December 2012; Revised: 25 February 2013; Accepted: 30 March 2013; Published online: 27 May 2013 
and demographics. Therefore, we conducted this study to explore the risk factors for HIV infection among MSM in Kunming, Yunnan Province, China. Our results may be generalised to other areas in China where different ethnic groups cohabit.

\section{MATERIALS AND METHODS}

A case-control study was conducted with a case-control ratio of $1: 4$. Cases and controls were matched by age ( \pm 3 years), and they were associated with the same group of MSM. Information on possible risk factors was collected by a survey questionnaire and a qualitative interview. Differences between the case and control groups were compared, and odds ratios (ORs) were calculated.

\section{Ethics statement}

We strictly followed the principles of respect, voluntary participation and confidentiality. Participants provided informed consent, and interviews were confidential. Participants could refuse to participate or exit the study at any time.

\section{Recruitment of cases and controls}

Participants were recruited from Yunnan Rainbow Sky, a communitybased organisation that specialises in HIV counselling, testing, and intervention services for MSM. Cases were confirmed as HIV-positive by the AIDS Department of Yunnan Centers for Disease Control and Prevention; MSM who tested HIV negative served as controls.

\section{Sample size estimation}

This research adopted non-probability sampling and respondentdriven sampling. According to the experience of Yunnan Rainbow Sky, approximately 30 MSM test HIV positive in a one-year period. To ensure the appropriate power of the statistical analysis, we aimed for 50 cases and 200 controls, for 250 samples in total.

\section{Questionnaires}

The design of the questionnaire was based on the MSM Health Questionnaire, which was obtained from the Implementation scheme manual of AIDS sentinel surveillance of China (2011). ${ }^{24}$ In addition, risk factors for HIV infection among MSM were added from the domestic and international literature. The questionnaire contents included demographic information, HIV/AIDS knowledge, MSM sexual behaviour, heterosexual behaviour and other relevant risk factors for HIV infection. The survey questionnaire was piloted and revised. All MSM approached participated in this study. Interviews were conducted one-on-one and face-to-face. Each interview lasted approximately $30 \mathrm{~min}$.

\section{Statistics}

EpiData 3.0 was used to create the database. Data were double entered for consistency. Data were analysed with SPSS17.0. We used a layered chi-square test and a multivariate analysis, applying Cox regression and conditional logistic regression models, and considered an $\alpha=0.05$ to indicate statistical significance.

\section{RESULTS}

\section{Sociodemographic characteristics of the study population}

There were 250 samples in total; 50 (20\%) men served as cases, and 200 $(80 \%)$ served as controls. The median age of the respondents was 30 $(29.68 \pm 8.77)$ years, with a range of 16 to 55 years. The age at the first sexual encounter with a man was between 12 and 50 years, with an average age of $22(21.96 \pm 6.10)$ years. Additionally, 167 (66.8\%) were unmarried, 42 (16.8\%) were married, 18 (7.2\%) cohabitated and 23 (9.2\%) were divorced or widowed. Of the participants, $206(82.4 \%)$ were Han, and $44(17.6 \%)$ were other ethnicity; $183(73.2 \%)$ were from Yunnan, and 67 (26.8\%) were from other provinces. The number of respondents with an education level of college or above was 118 $(47.2 \%)$. The numbers of respondents with education levels of high school and junior high school were similar at $58(23.2 \%)$ and 51 (20.4\%), respectively. By contrast, an elementary school education level and illiteracy were uncommon at $16(6.4 \%)$ and $7(2.8 \%)$, respectively. Commercial services was the most common profession with $83(33.2 \%)$ men, followed by $39(15.6 \%)$ students, $35(14.0 \%)$ cadre/staff, $30(12.0 \%)$ workers and $26(10.4 \%)$ house workers. The number of respondents in other professions was low. Most respondents $(65 ; 26.0 \%)$ had average monthly income between 2001 and 3000 Yuan, while $30(12.0 \%)$ respondents had an income greater than 4000 Yuan, and $18(7.2 \%)$ had an income of 1000 Yuan or less (Table 1).

\section{Univariate analyses of risk factors for HIV infection}

Univariate analyses indicated that the risk factors for HIV infection among MSM were primarily related to sexual behaviour, including an average monthly income of 2001-3000 Yuan ( $\mathrm{OR}=3.100, P<0.01)$, always drinking alcohol before sexual encounters $(O R=1.225$, $P<0.01)$, receptive anal sex $(\mathrm{OR}=1.161, P<0.01)$, insertive anal sex $(\mathrm{OR}=0.031, P<0.01)$, sometimes used condoms during anal sex in the last 6 months $(\mathrm{OR}=1.379, P<0.01)$, an HIV-positive sex partner $(\mathrm{OR}=5.306, P<0.01)$, rectal trauma with bleeding $(\mathrm{OR}=2.848$, $P<0.01)$, did not use condoms at last sexual encounter $(\mathrm{OR}=1.568$, $P<0.01)$, engaged in commercial sex $(\mathrm{OR}=5.444, P<0.051)$, did not use condoms at last commercial sexual encounter with men $(\mathrm{OR}=1.351, P<0.01)$, had anal sex less than 10 times in the last month $(\mathrm{OR}=0.636, P<0.01)$ and more than 16 sex partners in the last 6 months $(\mathrm{OR}=2.000, P<0.01)$ (Table 2$)$.

Factors such as profession, ethnicity, education level, HIV/AIDS knowledge, where the MSM met their sex partners, sexually transmitted disease (STI), hepatitis $\mathrm{C}$ virus infection and circumcision status were not significant in univariate analyses $(P>0.05)$.

\section{Multivariate logistic regression analysis of HIV infection}

The multivariate analysis used a logistic regression analysis and included variables with $\alpha=0.05$ in the univariate analyses, and the following variables were entered into the equation: average monthly income between 2001 and 3000 Yuan $(P<0.05$, OR=6.341), sometimes used condoms during anal sex in the last 6 months $(P<0.01$, $\mathrm{OR}=7.601)$, an HIV-positive sex partner $(P<0.01, \mathrm{OR}=5.273)$, rectal trauma with bleeding in the last 6 months $(P<0.01, \mathrm{OR}=2.947)$, did not use condoms at last sexual encounter $(P<0.05, \mathrm{OR}=1.278)$, engaged in commercial sex $(P<0.05, \mathrm{OR}=5.925)$ and more than 16 sex partners in the last 6 months $(P<0.05, \mathrm{OR}=1.175)$. These factors had an $\mathrm{OR}>1$ and can be considered risk factors for HIV infection among MSM. In addition, anal sex less than 10 times in the last one month $(P<0.05, \mathrm{OR}=0.002)$ and insertive anal sex $(P<0.05$, $\mathrm{OR}=0.116)$ had an $\mathrm{OR}<1$ and can be considered protective factors for HIV infection among MSM (Table 3).

\section{DISCUSSION}

This study shows that an average monthly income between 2001 and 3000 Yuan is a risk factor for HIV infection among MSM $(\mathrm{OR}>1)$. An average monthly income between 2001 and 3000 Yuan is considered a low to medium level income in Yunnan Province. This suggests that 
Table 1 Sociodemographic data

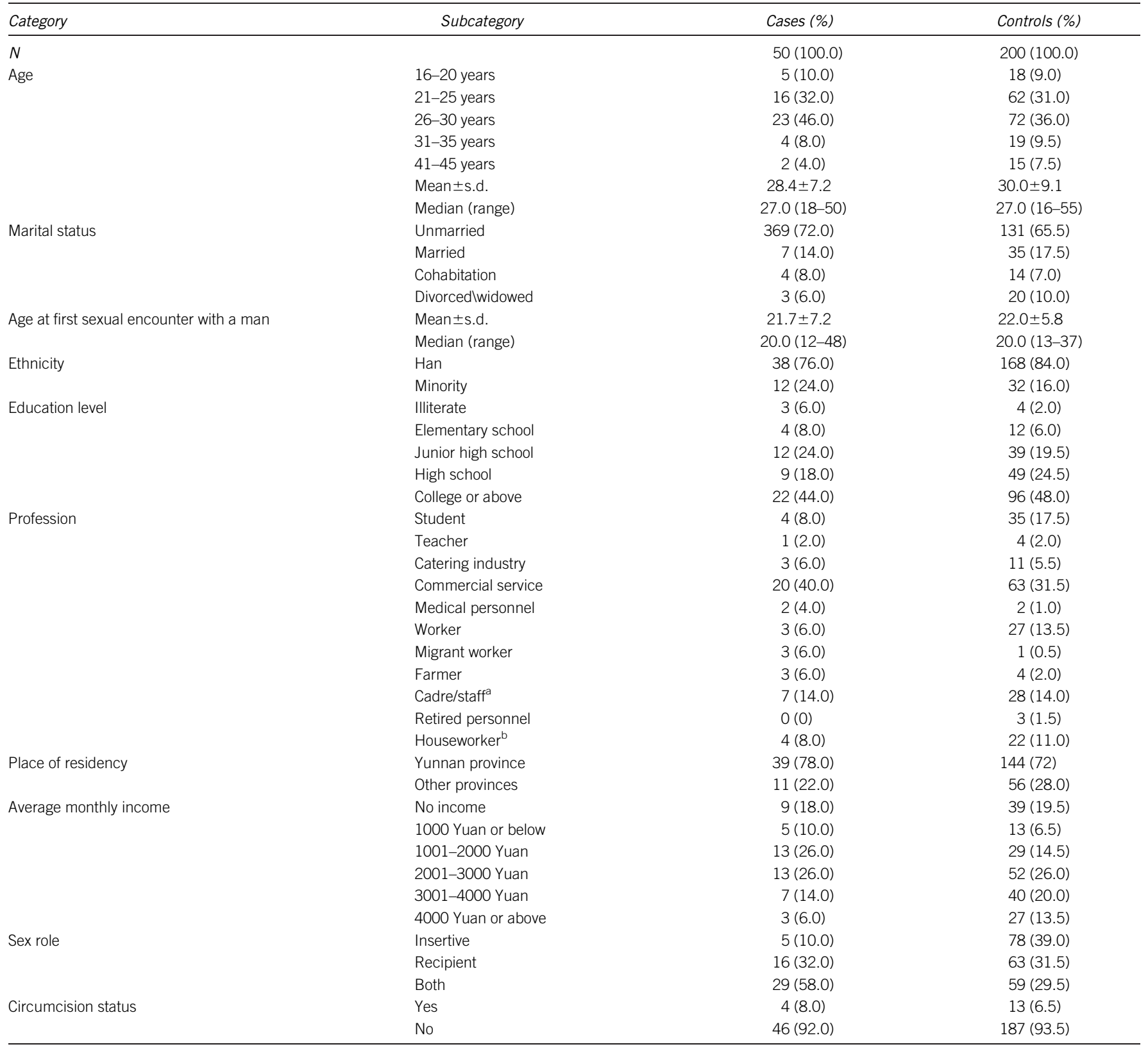

${ }^{a}$ Cadre/staff refers to state government functionaries.

${ }^{b}$ Housework Labourers refers to those with no job, who perform the housework at their own residence.

Table 2 Univariate analysis of risk factors for HIV infection

\begin{tabular}{|c|c|c|c|c|c|c|}
\hline Factors & Cases (\%) & Controls (\%) & $\chi^{2}$ & $P$ & Odds ratio & $95 \% \mathrm{Cl}$ \\
\hline Average monthly income 2001-3000 Yuan & $13(26.0)$ & $52(26.0)$ & 12.308. & 0.000 & 3.100 & $1.037-7.228$ \\
\hline Always drinks alcohol before sexual encounters & $14(28.0)$ & $14(7.0)$ & 18.106 & 0.000 & 1.225 & $1.109-2.634$ \\
\hline Receptive anal sex & $16(32.0)$ & $63(31.5)$ & 19.366 & 0.000 & 1.161 & $1.003-2.954$ \\
\hline Insertive anal sex & 5 (10.0) & $78(39.0)$ & 9.094 & 0.000 & 0.031 & $0.000-0.180$ \\
\hline Sometimes used condoms when having anal sex in the last 6 months & $38(76.0)$ & $98(49.0)$ & 23.257 & 0.000 & 1.379 & $1.113-2.881$ \\
\hline HIV-positive sex partner & $10(20.0)$ & $9(4.5)$ & 8.191 & 0.004 & 5.306 & $2.026-13.896$ \\
\hline Rectal trauma with bleeding in the last 6 months & $22(44.0)$ & $41(20.5)$ & 7.822 & 0.005 & 2.848 & $1.463-5.545$ \\
\hline Did not use condoms at last sexual encounter & $28(56.0)$ & $62(31.0)$ & 9.324 & 0.002 & 1.568 & $1.132-2.173$ \\
\hline Engaged in commercial sex & $5(10.0)$ & $4(2.0)$ & 5.796 & 0.016 & 5.444 & $1.406-21.088$ \\
\hline Had anal sex less than 10 times in the last one month & $4(8.3)$ & $52(28.9)$ & 210.457 & 0.000 & 0.636 & $0.407-0.995$ \\
\hline More than 16 sex partners in the last 6 months & $3(6.0)$ & $3(1.5)$ & 29.646 & 0.002 & 2.000 & $1.180-7.997$ \\
\hline Did not use condoms at last commercial sexual encounter with men & $3(50.0)$ & $0(0)$ & 5.091 & 0.024 & 1.351 & $1.100-2.043$ \\
\hline
\end{tabular}


Table 3 Multivariate logistic regression analysis of HIV infection

\begin{tabular}{|c|c|c|c|c|c|c|c|}
\hline Factors & Reference group & B & s.e. & Wald & $\mathrm{P}$ & $\operatorname{Exp}(\mathrm{B})$ & $95 \% \mathrm{Cl}$ \\
\hline Average monthly income 2001-3000 Yuan & Other income groups & 6.842 & 3.216 & 4.527 & 0.033 & 6.341 & $1.714-12.544$ \\
\hline HIV-positive sex partner & No HIV-positive sex partner & 1.663 & 0.618 & 7.249 & 0.007 & 5.273 & $1.572-17.691$ \\
\hline More than 16 sex partners in the last 6 months & Less than 16 sex partners in the last 6 months & 0.162 & 0.072 & 5.083 & 0.024 & 1.175 & $1.021-1.353$ \\
\hline Insertive anal sex & Recipient and both & -7.913 & 3.300 & 5.750 & 0.016 & 0.116 & $0.000-0.236$ \\
\hline $\begin{array}{l}\text { Sometimes used condoms }{ }^{a} \text { when having anal } \\
\text { sex in the last } 6 \text { months }\end{array}$ & Always used condoms & 7.105 & 2.385 & 8.873 & 0.003 & 7.601 & $1.359-23.083$ \\
\hline Engaged in commercial sex & Did not engage in commercial sex & 2.768 & 1.079 & 6.585 & 0.010 & 5.925 & $1.923-13.890$ \\
\hline
\end{tabular}

a 'Sometimes used condoms' refers to MSM who did not insist on condom use during sexual encounters. This is compared to 'always used condoms' and 'never used condoms'. 'Always used condoms' refers to MSM who correctly used condoms during every sexual encounter. 'Never used condoms' refers to MSM who never used condoms during sexual encounters.

MSM who have a lower income are more vulnerable to HIV infection. Inconsistent condom use is also a risk factor. Average income was not significant in the univariate analysis but was significant in the multivariate analysis. This is likely due to interactions between education level, profession and income. These interactions need to be investigated. Both sometimes used condoms during anal sex in the last 6 months and did not use condoms at last sexual encounter were associated with HIV infection $(\mathrm{OR}>1)$. A low frequency of condom use among MSM is a problem in many countries. In Nigeria, only $53 \%$ of MSM reported using condoms during anal sex, ${ }^{6}$ and in Nicaragua, only $30 \%$ MSM reported using condoms. ${ }^{25}$

More than 16 sex partners in the last 6 months and engaged in commercial sex were also risk factors for HIV infection $(\mathrm{OR}>1)$. These two factors may be related because MSM who engage in commercial sex are likely to have many sex partners. Other research has shown that having more than six sex partners is a risk factor for HIV infection among MSM $(\mathrm{OR}=12.95){ }^{26}$ In Thailand, $50.4 \%$ of MSM and transsexual women exchanged sex for money. Low education and monthly income were the primary reasons for engaging in commercial sex. ${ }^{27}$

A frequency of anal sex less than 10 times in the last month had an $\mathrm{OR}<1$ in both the univariate and multivariate analyses, indicating that a low frequency of anal sex is a protective factor for MSM. Insertive anal sex $(\mathrm{OR}<1)$ was also a protective factor against HIV infection among MSM, while receptive anal sex $(\mathrm{OR}=1.161)$ was a risk factor. This is likely due to the lower rate of HIV infection for insertive rather than receptive anal sex.

Always drinking alcohol before a sexual encounter was a risk factor $(\mathrm{OR}=1.225)$ in the univariate analysis. However, it was not significant in the multivariate analysis, perhaps because the individual role of drinking alcohol in HIV infection is stronger than its joint role. Large amounts of alcohol may lead to forgetting to use condoms and other problems, increasing the risk for infection. Alcohol dependence has been reported in China and India, but methamphetamine dependence has been reported in other countries. In the United States, approximately $96 \%(n=438)$ of MSM reported that methamphetamine use increased feelings of anger and decreased emotional support. ${ }^{28}$

However, where MSM met sex partners, particularly the Internet, ${ }^{29-30}$ age at first sexual encounter with a man, sexual masochism, ${ }^{31}$ STIs, hepatitis C virus infection, HIV/AIDS knowledge and other factors reported in China and abroad were not significant in this study and warrant further investigation. In particular, some reports have noted that STIs may be a risk factor for HIV infection among MSM, but our study did not observe this relationship. However, the proportion of participants with STIs was small. ${ }^{32,33}$
In addition, male circumcision (MC) has been shown to reduce the risk of HIV transmission. The World Health Organization and United Nations, Acquired Immune Deficiency Syndrome jointly recommended that the international community consider MC as an HIV prevention measure. ${ }^{34}$ However, male circumcision is not accepted in China. The reasons for the refusal of MC among Chinese men include the following: not necessary or not effective, $81.1 \%$ (997/1230); potential danger associated with surgery, $13.2 \%$ (162/1230); concern about reducing sexual ability, $10.4 \%$ (128/1230); and concern about cost of surgery, 5.7\% (70/1230). ${ }^{35}$ We suggest that more health education campaigns should focus on the positive health effects of MC to increase the MC rate in China.

Meanwhile, we collected data on participants' previous exposure. However, information bias cannot be excluded because the information we collected is sensitive, and participants may not have responded truthfully. This study adopted non-probability sampling, and the samples may therefore not be representative of the population. Due to these limitations, causal inferences have to be drawn with caution.

\section{CONCLUSION}

This study demonstrates that a low to medium income, inconsistent condom use, having an HIV-positive sex partner, rectal trauma with bleeding, engaging in commercial sex, and more than 16 sex partners in the last 6 months are risk factors for HIV infection among MSM. In addition, a low frequency of anal sex ( $<10$ times in the last month) was a protective factor against HIV infection among MSM. Meanwhile, the type of sexual behaviour influenced the rate of HIV infection; compared with insertive anal intercourse, receptive anal intercourse was more likely to result in HIV infection. Interventions should be targeted at MSM whose average monthly income is between 2001 and 3000 Yuan and those who engage in commercial sex. In addition, the importance of using condoms at every sexual encounter should be emphasised in health education, as should the treatment of rectal trauma with bleeding. Finally, MSM should decrease the number of sex partners and frequency of anal sex to decrease the rate of HIV infection.

\section{COMPETING FINANCIAL INTERESTS}

All authors declare that there are no competing financial interests.

\section{ACKNOWLEDGMENTS}

We would like to acknowledge the Yunnan Centers for Disease Control and Prevention's support regarding the included items and the foundation of the 
study. Meanwhile, we sincerely thank all the MSM who participated in this study.

1 Moore RD. Epidemiology of HIV in fection in the united states: implications for linkage to care. Clin Infect Dis 2011; 52: 208-13.

2 Centers for Disease Control and Prevention (CDC). Prevalence and awareness of HIV infection among men who have sex with men-21 cities, United States, 2008. MMWR Morb Mortal Wkly Rep 2010; 59: 1201-7.

3 WHO, UNICEF, UNAIDS. Progress report 2011: Global HIV/AIDS response. http:// www.who.int/hiv/pub/progress_report2011/en/index.html (accessed 30 November 2011)

4 Ministry of Health of the People's Republic of China, UNAIDS, WHO. Working Report on China's AIDS Epidemic Estimate in 2009. Beijing: Ministry of Health of the People's Republic of China; 2010.

5 She M, Zhang HB, Wang J, Xu J, Duan YW et al. Investigation of HIV and syphilis infection status and risk sexual behavior among men who have sex with men in four cities of China. Zhonghua Yu Fang Yi Xue Za Zhi 2012; 46: 324-8.

6 Strömdahl S, Onigbanjo Williams A, Eziefule B, Emmanuel G, Iwuagwu S et al. Associations of consistent condom use among men who have sex with men in Abuja, Nigeria. AIDS Res Hum Retroviruses 2012; 28: 1756-62.

7 Jenness SM, Neaigus A, Murrill CS, Gelpi-Acosta C, Wendel T et al. Recruitmentadjusted estimates of HIV prevalence and risk among men who have sex with men: effects of weighting venue-based sampling data. Public Health Rep 2011; 126: 63542.

8 Jenness SM, Neaigus A, Murrill CS, Gelpi-Acosta C, Wendel T et al. Recruitmentadjusted estimates of HIV prevalence and risk among men who have sex with men: effects of weighting venue-based sampling data. Public Health Rep 2011; 126: 63542.

9 Colón-López V, Rodríguez-Díaz CE, Ortiz AP, Soto-Salgado M, Suárez E et al. HIVrelated risk behaviors among a sample of men who have sex with men in Puerto Rico: an overview of substance use and sexual practices. PR Health Sci J 2011; 30: 65-8.

10 Rosenberg ES, Sullivan PS, Dinenno EA, Salazar LF, Sanchez TH. Number of casual male sexual partners and associated factors among men who have sex with men: results from the National HIV Behavioral Surveillance system. BMC Public Health $2011 ; 11: 189$.

11 Ghanem A, Little SJ, Drumright L, Liu L, Morris S et al. High-risk behaviors associated with injection drug use among recently HIV-infected men who have sex with men in San Diego, CA. AIDS Behav 2011; 15: 1561-9.

12 Mustanski B, Newcomb ME, Clerkin EM. Relationship characteristics and sexual risktaking in young men who have sex with men. Health Psychol 2011: 30: 597-605.

13 Safren SA, Reisner SL, Herrick A, Mimiaga MJ, Stall R. Mental health and HIV risk in men who have sex with men. J Acquir Immune Defic Syndr2010; 55(Suppl 2): s74-7.

14 Mehta SA, Silvera R, Bernstein K, Holzman RS, Aberg JA et al. Awareness of postexposure HIV prophylaxis in high-risk men who have sex with men in New York City. Sex Transm Infect 2011; 87: 344-8.

15 Rispel LC, Metcalf CA, Cloete A, Reddy V, Lombard C. HIV prevalence and risk practices among men who have sex with men in two South African cities. J Acquir Immune Defic Syndr 2011; 57: 69-76.

16 Guy RJ, Spelman T, Stoove M, El-Hayek C, Goller J et al. Risk factors for HIV seroconversion in men who have sex with men in Victoria, Australia: results from a sentinel surveillance system. Sex Health 2011; 8: 319-29.
17 Baral S, Adams D, Lebona J, Kaibe B, Letsie P et al. A cross-sectional assessment of population demographics, HIV risks and human rights contexts among men who have sex with men in Lesotho. J Int AIDS Soc 2011; 14: 36.

18 Zhou C, Ding XB, Liu F. Investigation on HIV/AIDS related konwledge and behavior among men who have sex with men. Mod Prev Med 2010; 37: 763-5.

19 UNAIDS. Monitoring the Declaration of Commitment on HIV/AIDS: Guidelines on Construction of Core Indicators: 2010 Reporting. Geneva: UNAIDS; 2010.

20 Mimiaga MJ, Thomas B, Mayer KH, Reisner SL, Menon S et al. Alcohol use and HIV sexual risk among MSM in Chennai, India. Int J STD AIDS 2011; 22: 121-5.

21 Malhotra N, Yang J. Risky behaviour and HIV prevalence among Zambian men. J Biosoc Sci. 2011; 43: 155-65.

22 Parry C, Petersen P, Dewing S, Carney T, Needle R et al. Rapid assessment of drugrelated HIV risk among men who have sex with men in three South African cities. Drug Alcohol Depend 2008; 95: 45-53.

23 Hao C, Yan H, Yang H, Huan X, Guan W et al. The incidence of syphilis, HIV and HCV and associated factors in a cohort of men who have sex with men in Nanjing, China. Sex Transm Infect 2011; 87: 199-201.

24 Chinese Centre for Disease Control and Prevention, National Centre for AIDS/STD Control and Prevention. Implementation Scheme Manual of AIDS Sentine Surveillance of China. Beijing: People's Medical Publishing House; 2011. pp11-2.

25 Ugarte Guevara WJ, Valladares Cardoza E, Essén B. Sexuality and risk behavior among men who have sex with men in León, Nicaragua: a mixed methods approach. J Sex Med 2012; 9: 1634-48.

26 She M, Zhang HB, Wang J, Xu J, Duan YW et al. Investigation of HIV and syphilis infection status and risk sexual behavior among men who have sex with men in four cities of China. Zhonghua Yu Fang Yi Xue Za Zhi 2012; 46: 324-8.

27 Newman PA, Lee SJ, Roungprakhon S, Tepjan S. Demographic and behavioral correlates of HIV risk among men and transgender women recruited from gay entertainment venues and community-based organizations in Thailand: implications for HIV prevention. Prev Sci 2012; 13: 483-92.

28 Semple SJ, Strathdee SA, Zians J, Patterson TL. Factors associated with experiences of stigma in a sample of HIV-positive, methamphetamine-using men who have sex with men. Drug Alcohol Depend 2012; 125: 154-9.

29 Lim SH, Guadamuz TE, Wei C, Chan R, Koe S. Factors Associated with unprotected receptive anal intercourse with internal ejaculation among men who have sex with men in a large Internet sample from Asia. AIDS Behav 2012; 16: 1979-87.

30 White JM, Mimiaga MJ, Reisner SL, Mayer KH. HIV sexual risk behavior among black men who meet other men on the Internet for sex. J Urban Health; e-pub ahead of print 12 June 2012; doi:10.1007/s11524-012-9701-y.

31 Lloyd S, Operario D. HIV risk among men who have sex with men who have experienced childhood sexual abuse: systematic review and meta-analysis. AIDS Educ Prev2012; 24: $228-41$

32 Mor Z, Shohat T, Goor Y, Dan M. Risk behaviors and sexually transmitted diseases in gay and heterosexual men attending an STD clinic in Tel Aviv, Israel: a cross-sectional study. Isr Med Assoc J 2012; 14: 147-51.

33 Yin YP, Chen SC, Wang HC, Wei WH, Wang QQ et al. Prevalence and risk factors of HSV-2 infection and HSV-2/HIV coinfection in men who have sex with men in China: a multisite cross-sectional study. Sex Transm Dis 2012; 39: 354-8.

34 Srithanaviboonchai K, Grimes RM. Why Thailand should consider promoting neonatal circumcision? Southeast Asian J Trop Med Public Health 2012; 43: 1218-26.

35 Yang X, Abdullah AS, Wei B, Jiang J, Deng W et al. Factors influencing Chinese male's willingness to undergo circumcision: a cross-sectional study in Western China. PLoS ONE 2012; 7: e30198. 